\title{
Photoluminescence from ultrathin Ge-rich multiple quantum wells observed up to room temperature: Experiments and modeling
}

\author{
T. Wendav, ${ }^{1, *}$ I. A. Fischer, ${ }^{2}$ M. Virgilio, ${ }^{3}$ G. Capellini,,${ }^{4,5}$ F. Oliveira, ${ }^{6}$ M. F. Cerqueira, ${ }^{6}$ A. Benedetti, ${ }^{7}$ S. Chiussi, ${ }^{8}$ \\ P. Zaumseil, ${ }^{4}$ B. Schwartz, ${ }^{9}$ K. Busch, ${ }^{1,10}$ and J. Schulze ${ }^{2}$ \\ ${ }^{1}$ AG Theoretische Optik \& Photonik, Humboldt-Universität zu Berlin, Newtonstr. 15, D-12489 Berlin, Germany \\ ${ }^{2}$ Institut für Halbleitertechnik, Universität Stuttgart, Pfaffenwaldring 47, D-70569 Stuttgart, Germany \\ ${ }^{3}$ Dipartimento di Fisica "E. Fermi”, Università di Pisa, L.go Pontecorvo 3, I-56127 Pisa, Italy \\ ${ }^{4}$ IHP, Im Technologiepark 25, D-15236 Frankfurt (Oder), Germany \\ ${ }^{5}$ Dipartimento di Scienze, Università Roma Tre, Viale Marconi 446, I-00146 Roma, Italy \\ ${ }^{6}$ Centre of Physics, University of Minho, Campus de Gualtar, 4710-057 Braga, Portugal \\ ${ }^{7}$ CACTI, Universidade de Vigo, Campus Universitario, 36310 Vigo, Spain \\ ${ }^{8}$ Departamento de Física Aplicada, Universidade de Vigo, Campus Universitario, 36310 Vigo, Spain \\ ${ }^{9}$ Institut für Physik, Brandenburgische Technische Universität Cottbus-Senftenberg, Platz der Deutschen Einheit 1, \\ D-03046 Cottbus, Germany \\ ${ }^{10}$ Max-Born-Institut, Max-Born-Str. 2A, D-12489 Berlin, Germany
}

(Received 20 September 2016; revised manuscript received 8 November 2016; published 8 December 2016)

\begin{abstract}
Employing a low-temperature growth mode, we fabricated ultrathin $\mathrm{Si}_{1-x} \mathrm{Ge}_{x} / \mathrm{Si}$ multiple quantum well structures with a well thickness of less than $1.5 \mathrm{~nm}$ and a Ge concentration above $60 \%$ directly on a Si substrate. We identified an unusual temperature-dependent blueshift of the photoluminescence (PL) and exceptionally low thermal quenching. We find that this behavior is related to the relative intensities of the no-phonon (NP) peak and a phonon-assisted replica that are the main contributors to the total PL signal. To investigate these aspects in more detail, we developed a strategy to calculate the PL spectrum employing a self-consistent multivalley effective mass model, in combination with second-order perturbation theory. Through our investigation, we find that while the phonon-assisted feature decreases with temperature, the NP feature shows a strong increase in the recombination rate. Besides leading to the observed robustness against thermal quenching, this causes the observed blueshift of the total PL signal.
\end{abstract}

DOI: 10.1103/PhysRevB.94.245304

\section{INTRODUCTION}

While group IV elemental semiconductors $\mathrm{Si}$ and $\mathrm{Ge}$ and their $\mathrm{Si}_{1-x} \mathrm{Ge}_{x}$ alloys dominate semiconductor electronics, their use in optoelectronics is limited due to their indirect fundamental band gap and the consequently poor radiative recombination efficiency [1]. Therefore, nanostructures based on the SiGe system have been considered as a potentially active material class with improved optical performance [2]. In particular, $\mathrm{Si}_{1-x} \mathrm{Ge}_{x}$ quantum wells $(\mathrm{QWs})$ have been the subject of theoretical and experimental investigations [3,4]. Among other techniques, photoluminescence (PL) has proven to be a particularly useful method for studying optical transitions in such structures [5-8]. Shortly following the first PL investigations of SiGe/Si single and multiple QWs by Noël et al. [9], the first well-resolved low-temperature band edge PL was observed by Sturm et al. [10], who attributed the observed PL spectral features to type II transitions between holes that are localized in the Ge-rich well regions and electrons that are localized in the Si barrier layers occurring at the barrier-well interface. After the successful demonstration of modulation based on the quantum-confined Stark effect in strained Ge QWs sandwiched between Ge-rich barrier layers and embedded in PIN diodes [11,12], similar SiGe/Ge multiple-QW structures have been used to demonstrate direct band gap PL originating from type I transitions within the Ge

\footnotetext{
*Corresponding author: wendav@physik.hu-berlin.de
}

wells [13]. Finally, there is ongoing interest in designing $\mathrm{SiGe}$ QW structures for intraband transitions for applications not only in QW infrared (IR) photodetectors [14-17] but also in quantum cascade laser structures [18].

One of the main challenges in utilizing SiGe-based QW structures for optical device applications is the thermal quenching of their luminescence. In most experiments on $\mathrm{SiGe} / \mathrm{Si}$ QWs, PL spectra can only be observed at temperatures below a few tens of degrees Kelvin [19,20]. The thermal PL quenching is mainly because of nonradiative recombination centers [19] that can originate, e.g., from structural defects such as dislocations. Higher growth temperatures have been shown to reduce thermal quenching [7,19]; however, they also lead to $\mathrm{Si}$ and $\mathrm{Ge}$ interdiffusion and thus to the broadening of heterointerfaces [21]. Although room temperature PL has been reported in Ge QWs sandwiched between $\mathrm{Si}_{1-x} \mathrm{Ge}_{x}$ barriers [13], those structures have the disadvantage of requiring a relatively thick $\mathrm{SiGe}$ virtual substrate to accommodate the large lattice mismatch between the Si substrate and the QW structures.

Here, we report the room temperature PL emission of ultrathin SiGe wells featuring very high Ge content grown directly on $\mathrm{Si}$, without using a virtual substrate technology. Our growth strategy consisted of repeatedly depositing 5.5 monolayers (ML) of Ge and overgrowing them with $\mathrm{Si}$ at low growth temperatures. We find that this enables us to fabricate a multiple-QW structure containing $10 \mathrm{SiGe}$ QWs with a $\mathrm{Ge}$ content exceeding $60 \%$. The high quality of the layers is confirmed by a structural analysis based on high-resolution transmission electron microscopy (HR-TEM), as well as x-ray 
diffraction (XRD). To interpret PL spectra, we developed a strategy to simulate the PL for the sample under investigation based on a coupled Schrödinger-Poisson description in the effective mass approximation of the electronic states belonging to different near-gap valleys. Even though the PL of various $\mathrm{SiGe/Si} \mathrm{QW}$ structures has been extensively investigated experimentally, theoretical calculations of the PL spectrum based on an effective mass approach have not been reported in the literature. In papers comparing measured PL spectra with theoretical calculations, the PL transition energies are commonly computed by taking into account the topmost valence and bottommost conduction states only [22,23]. This can be helpful in cases of low optical excitation and temperature. However, for higher temperatures and stronger excitations, the PL shape strongly depends on the filling of the bands due to the optically excited excess carriers and on the temperature-dependent quasi-Fermi distributions of the excited charge carriers, which leads to the population of higher energy subband states, as well as to relevant band bending effects.

In the analysis reported here, we describe the features from indirect recombination between quantum confined states relying on second-order perturbation theory, which, to our knowledge, has not been reported elsewhere in the literature. For second-order perturbative absorption analysis in two-dimensional (2D) semiconductors, see, for instance, Refs. [24,25].

Our manuscript is organized as follows. We describe the sample growth and the experimental methods for its analysis in Sec. II. In Sec. III, we introduce the theoretical method used for the calculation of the PL spectra. Sec. IV is divided into two parts. In the first part, the experimental results concerning the structural properties of the sample are described. In the second part, we present the PL measurements. We start with the excitation-density-dependent measurements, and we compare experimental and simulated spectra to establish the validity of our method. In a second step, we apply our theoretical method to the temperature-dependent measurements. We conclude our analysis in Sec. V by discussing the physical consequences of our findings.

\section{SAMPLE PREPARATION AND EXPERIMENTAL SETUP}

The nominally intrinsic Ge multiple-QW sample was fabricated by solid-source molecular beam epitaxy (MBE) on a Si (100) substrate. After thermal desorption of the native oxide, a 100 -nm-thick Si buffer layer was grown at $600{ }^{\circ} \mathrm{C}$. A 10 period sequence in which each period consists of $5.5 \mathrm{ML}$ of $\mathrm{Ge}$ and $10 \mathrm{~nm}$ of $\mathrm{Si}$ (Fig. 1) was grown at a constant growth temperature of $350^{\circ} \mathrm{C}$. The Ge layers were grown at a rate of $0.087 \AA / \mathrm{s}$, while for the Si spacer layers, a growth rate of $1 \AA$ / s was used. A JEOL JEM2010F microscope was used for TEM characterization. Raman scattering experiments were performed at room temperature in a backscattering geometry on an Alpha300 R confocal Raman microscope (WITec), using a diode-pumped solid-state laser with a wavelength of $532 \mathrm{~nm}$ as an excitation source. The spot size on the sample was roughly $1.4 \mu \mathrm{m}^{2}$, with a power of roughly $4 \mathrm{~mW}$ measured close to the external sample surface. The XRD measurements were carried out

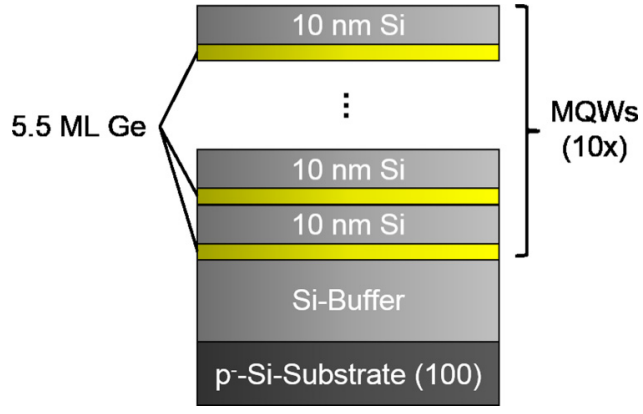

FIG. 1. Schematic of $\mathrm{SiGe/Si}$ multiple-QW sample stack sequence.

with a SmartLab diffractometer from Rigaku using $\mathrm{CuK}_{\alpha}$ radiation. Last, microphotoluminescence ( $\mu \mathrm{PL})$ measurements were carried out at lattice temperatures varying between 80 and $300 \mathrm{~K}$ using a custom-designed Horiba setup featuring a $50 \times$ optical microscope (numerical aperture $=0.65$ ), a high-resolution spectrometer optimized for IR measurements (Horiba iHR320), and an extended-InGaAs detector (0.6 to $1.1 \mathrm{eV}$ detection range). A $532 \mathrm{~nm}$ laser with an output optical power between 0.5 and $23 \mathrm{~mW}$ was focused on the sample surface, with an excitation power density ranging between $3.2 \times 10^{4}$ and $160 \times 10^{4} \mathrm{Wcm}^{-2}$. All spectra were collected at normal incidence in backscattering geometry, and a white-body lamp was used to determine the optical response of the setup used for the calibration.

\section{NUMERICAL MODEL}

To better understand the observed PL, we compare experimental spectra with numerical simulations. As a first step, we compute the electronic states, relying on a multivalley Schrödinger-Poisson code. Spectrally resolved recombination rates, associated with band-to-band indirect transitions mediated by electron-phonon scattering, are then calculated in the framework of second-order perturbation theory. We consider a 2D carrier interacting with a three-dimensional (3D) bulk phonon bath. Since our ultrathin multiple-QW samples feature type II band alignment, the eigenstates are sensitive to the amount of pump-induced excess carrier density in the well. However, this quantity cannot be easily estimated theoretically. Therefore, in our simulative approach, we chose to phenomenologically relate the excess carrier density to the pump power, introducing a fitting constant. Its value is set by calibrating numerical data for the pump-induced blueshift of the PL peak against experimental data. This blueshift is controlled by the band bending related to electrostatic fields, caused by the spatial separation of the photoexcited electrons and holes. It follows that its magnitude can be used to indirectly estimate the amount of pump-induced excess carrier density in the samples.

For the calculation of PL spectra, we are interested in interband radiative transitions involving the quantum-confined subband states associated with different near-gap valence and conduction valleys. In this regard, the type II band alignment between well and barrier regions is important for the considered SiGe/Si multiple-QW structure. The type II 
band alignment between well and barrier regions results in the spatial separation of photoexcited electrons and holes, as well as power-dependent band bending effects, which significantly influence the transition energies. These issues, together with the splitting and shifts of bands due to the biaxial strain affecting the $\mathrm{SiGe}$ region, have been addressed by solving the Schrödinger-Poisson equation in effective mass approximation for the electronic states belonging to different near-gap valleys.

In our model, we have considered heavy hole $(\mathrm{HH})$, light hole (LH), and split-off (SO) bands in the valence band and $\Gamma_{c}, L, \Delta_{2}$ and $\Delta_{4}$ valleys in the conduction band. The $\mathrm{SiGe} / \mathrm{Si} \mathrm{QW}$ composition profile is obtained from experimental data, and periodic boundary conditions were applied. Strain effects on the band edge and valence masses are calculated assuming coherent growth and relaxed Si layers as described in Refs. [26,27]. For a given pump-induced excess carrier density, valence and conduction quasi-Fermi energies and band bending are self-consistently evaluated, taking into account the 2D density of states resulting from all considered valleys.

Following the computation of hole and electron eigenstates, the PL spectrum is calculated. In our $\mathrm{SiGe/Si} \mathrm{multiple-QW}$ sample, holes are confined in the compressively strained SiGe QW region and are distributed mainly in the HH1 subband and to a minor extent in the LH1 subband close to the $\Gamma$ point. However, electrons are mainly localized in the unstrained $\mathrm{Si}$ barrier region and are associated with the $\Delta$ valley. Therefore, a radiative recombination can happen only if the missing momentum is provided by either phonons (phonon-assisted recombination) or elastic scattering centers like crystal defects, alloy disorder, or interface roughness (NP recombination). The challenge in calculating the full PL spectrum is to estimate the relative intensity of the phonon-assisted and NP recombination rates. For the phonon-assisted process, the electron-phonon coupling can be estimated using the effective deformation potential for $\Delta-\Gamma$ scattering reported in the literature [28,29]; however, we do not have sufficient information on the structural properties of the sample to realistically calculate the elastic scattering rates because of the relevant role played by the heterointerface properties. Moreover, from a theoretical point of view, elastic interface roughness scattering effects in QW systems are commonly treated within a perturbative framework, since well thickness is typically one or two orders of magnitude larger than the interface region. However, due to the ultrathin layer thickness of the well region in our investigated samples, this approximation cannot be used. Therefore, in this paper, we focus on the calculation of the phonon-assisted PL contribution only, avoiding the estimation of the PL intensity ratio between the phonon and the NP features. Upon knowing the peak energy of the phonon-assisted recombination spectrum and the energy of the contributing phonons, we can estimate the energy of the NP feature.

To calculate the spectrally resolved emission rate of photons resulting from indirect band-to-band recombination, we used the second-order Fermi golden rule:

$$
P_{i \rightarrow f}=\frac{2 \pi}{\hbar}\left|\sum_{m} \frac{\left\langle i\left|H_{\mathrm{ph}}\right| m\right\rangle\left\langle m\left|H_{\mathrm{em}}\right| f\right\rangle}{E_{m}^{\prime}-E_{f}^{\prime}}\right|^{2} \delta\left(E_{i}^{\prime}-E_{f}^{\prime}\right),
$$

with the energies $E^{\prime}$ corresponding to the total energy of the states, including electronic, phononic, and photonic contributions. The initial states are the populated subbands of the $\Delta_{2}$ and $\Delta_{4}$ valleys (indicated by $\Delta_{c}$ ). The electronic final states are the populated subbands of the $\mathrm{HH}, \mathrm{LH}$, and SO bands (indicated by $\Gamma_{v}$ ). For energetic reasons, intermediate electronic states belong to the $\Gamma$ conduction band (indicated by $\Gamma_{c}$ ) only. Furthermore, due to the large confinement energy of conduction electrons in this band, only the lowest $\Gamma_{c}$ subband needs to be considered. This leads to the following definition of the states:

$$
\begin{aligned}
|i\rangle & =\left|\Psi_{\Delta_{c}}^{(l)}\left(\boldsymbol{k}_{\|}\right)\right\rangle|0\rangle\left|\ldots n_{\mathrm{ph}}(\boldsymbol{q}) \ldots\right\rangle \\
\left|m^{\mathrm{em} / \mathrm{abs}}\right\rangle & =\left|\Psi_{\Gamma_{c}}^{(1)}\left(\boldsymbol{k}_{\|}^{\prime}\right)\right\rangle|0\rangle\left|\ldots\left(n_{\mathrm{ph}}(\boldsymbol{q}) \pm 1\right) \ldots\right\rangle \\
\left|f^{\mathrm{em} / \mathrm{abs}}\right\rangle & =\left|\Psi_{\Gamma_{v}}^{(s)}\left(\boldsymbol{k}_{\|}^{\prime}\right)\right\rangle|0 \ldots 1 \omega \ldots\rangle\left|\ldots\left(n_{\mathrm{ph}}(\boldsymbol{q}) \pm 1\right) \ldots\right\rangle,
\end{aligned}
$$

where the factors of the product state represent the electronic, photonic, and phononic states of the system; $l$ and $s$ are conduction and valence subband indices, respectively; upper and lower signs refer to phonon emission (em) and absorption (abs), respectively; and $|0 \cdots 1 \omega \cdots\rangle$ indicates that a photon with energy $\hbar \omega$ has been emitted. The electronic part is given by

$$
\left\langle\boldsymbol{r} \mid \Psi_{\alpha}^{(l)}\left(\boldsymbol{k}_{\|}\right)\right\rangle=\frac{1}{\sqrt{A}} e^{i \boldsymbol{k}_{\|} \cdot \rho} \phi_{\alpha}^{(l)}(z) u_{\alpha}(\boldsymbol{r}),
$$

where $\phi_{\alpha}^{(l)}(z)$ is the envelope function of subband $l$ of valley $\alpha, u_{\alpha}$ is the related Bloch function, and $A$ is the area of cross section perpendicular to the growth direction. The interaction between electrons and bulk phonons is given by

$$
\begin{aligned}
H_{\mathrm{ph}}= & \sum_{\boldsymbol{q}_{\|}, q_{z}} \sum_{n, m} \sum_{k_{\|}} D_{\boldsymbol{q}_{\|}, q_{z}} M_{n, m}\left(q_{z}\right)\left(b_{\boldsymbol{q}_{\|}, q_{z}}+b_{-\boldsymbol{q}_{\|},-q_{z}}^{\dagger}\right) \\
& \times c_{n, \boldsymbol{k}_{\|}+\boldsymbol{q}_{\|}}^{\dagger} c_{m, \boldsymbol{k}_{\|}},
\end{aligned}
$$

where $n$ and $m$ label 2D electronic states. For simplicity, following Refs. [28,29], we approximate the electron-phonon interaction, considering only an effective dispersionless phonon branch at $\hbar \omega_{\text {ph }}=50 \mathrm{meV}$. This value has been set equal to the energy separation between the NP and the phonon-assisted spectral features measured experimentally. As one may expect, this energy is close to the transverse optical (TO) phonon energy of bulk $\mathrm{Si}(58.8 \mathrm{meV})[30]$ at the $X$ point. However, the Si-Ge vibrational mode has comparable energy $(50 \mathrm{meV})$ [30]; hence, it cannot be excluded that interaction with this latter vibrational mode may contribute to the phonon-assisted indirect transition rate. Since our spectral resolution does not allow us to resolve the two phonon channels, and numerically we are not interested in absolute values, we consider a single bulklike effective phonon mode in our model. For nonpolar lattices, one can assume

$$
D_{\boldsymbol{q}_{\|}, q_{z}}=D_{\mathrm{eff}} \sqrt{\frac{\hbar^{2}}{2 \rho V \hbar \omega_{\mathrm{ph}}}},
$$

where $\rho$ is the mass density, $V$ is the volume, and $D_{\text {eff }}$ is an effective deformation potential. The squared matrix element 
for the electron-phonon interaction is then given by

$$
\begin{aligned}
& \left|\left\langle i\left|H_{\mathrm{ph}}\right| m^{\mathrm{em} / \mathrm{abs}}\right\rangle\right|^{2} \\
& \quad=D_{\mathrm{eff}}^{2} \frac{\hbar^{2}}{2 \rho V \hbar \omega_{\mathrm{ph}}}\left(n_{\mathrm{ph}}+\frac{1}{2} \pm \frac{1}{2}\right)\left|M_{\Delta_{c}}^{(l)}\left(q_{z}\right)\right|^{2} .
\end{aligned}
$$

When considering $\Delta_{c}$ and $\Gamma_{c}$ for the initial and intermediate states, $M_{n, m}\left(q_{z}\right)$ can be written as

$$
M_{\Delta_{c}}^{(l)}\left(q_{z}\right)=\int_{-\frac{L}{2}}^{\frac{L}{2}}\left(\phi_{\Delta_{\mathrm{c}}}^{(l)}(z)\right)^{*} e^{i q_{z} z} \phi_{\Gamma_{c}}^{(1)}(z) d z,
$$

where $l$ is the subband index in the $\Delta_{c}$ valley and $L$ is the single well and barrier length.

The electromagnetic interaction is, according to the usual notation, given by

$$
H_{\mathrm{em}}=\frac{e}{m_{0}} \boldsymbol{A} \cdot \boldsymbol{p}=\frac{e}{m_{0}} \sqrt{\frac{\hbar}{2 V \omega \varepsilon_{0} \varepsilon}}\left(a_{\hat{\boldsymbol{e}}}+a_{\hat{\boldsymbol{e}}}^{\dagger}\right) \hat{\boldsymbol{e}} \cdot \boldsymbol{p},
$$

leading to the following expression of the squared interaction matrix element:

$$
\left|\left\langle m^{\mathrm{em} / \mathrm{abs}}\left|H_{\mathrm{em}}\right| f^{\mathrm{em} / \mathrm{abs}}\right\rangle\right|^{2}=\left(\frac{e}{m_{0}}\right)^{2} \frac{\hbar}{2 V \omega \varepsilon_{0} \varepsilon}\left|I_{\Gamma_{v}}^{(s)}\right|^{2}\left|\boldsymbol{p}_{c v}^{\Gamma} \cdot \hat{\boldsymbol{e}}\right|^{2}
$$

In the above expressions, $I_{\Gamma_{v}}^{(s)}$ and $\boldsymbol{p}_{c v}^{\Gamma}$ are defined as

$$
\begin{aligned}
& I_{\Gamma_{v}}^{(s)}=\int_{-L / 2}^{+L / 2}\left(\phi_{\Gamma_{c}}^{(1)}(z)\right)^{*} \phi_{\Gamma_{v}}^{(s)}(z) d z, \\
& \boldsymbol{p}_{c v}^{\Gamma}=\int_{\Omega} u_{\Gamma_{c}}^{*}(\boldsymbol{r}) \boldsymbol{p} u_{\Gamma_{v}}(\boldsymbol{r}) d^{3} \boldsymbol{r} .
\end{aligned}
$$

The spectrally resolved rate of spontaneous emission of photons via phonon-assisted recombinations can then be calculated by summing over all electronic and phononic degrees of freedom and polarization modes related to photons emitted normally to the sample surface. Considering the Fermi distributions $f_{e}\left(\boldsymbol{k}_{\|, i}\right)$ and $f_{h}\left(\boldsymbol{k}_{\|, f}\right)$ for electrons and holes, we obtain

$$
\begin{aligned}
R_{\mathrm{sp}}^{\mathrm{ind}}(\hbar \bar{\omega}) \mathrm{d}(\hbar \bar{\omega})= & \frac{1}{V} \sum_{\hat{\boldsymbol{e}}} \sum_{i, f} P_{i \rightarrow f} f_{e}\left(\boldsymbol{k}_{\|, i}\right) f_{h}\left(\boldsymbol{k}_{\|, f}\right) G(\hbar \omega) \delta(\hbar \omega-\hbar \bar{\omega}) d(\hbar \bar{\omega}) \\
= & F_{\text {ind }} \sum_{\hat{\boldsymbol{e}}} \sum_{i, f}\left(n_{\mathrm{ph}}+\frac{1}{2} \pm \frac{1}{2}\right)\left|I_{\Gamma_{v}}^{(s)}\right|^{2}\left|\boldsymbol{p}_{c v}^{\Gamma} \cdot \hat{\boldsymbol{e}}\right|^{2}\left|\sum_{q_{z}} M_{\Delta_{c}}^{(l)}\left(q_{z}\right)\right|^{2} \\
& \times f_{e}\left(\boldsymbol{k}_{\|, i}\right) f_{h}\left(\boldsymbol{k}_{\|, f}\right) \frac{\delta\left(E_{i}\left(\boldsymbol{k}_{\|, i}\right)-E_{f}\left(\boldsymbol{k}_{\|, f}\right) \mp \hbar \omega_{\mathrm{ph}}-\hbar \bar{\omega}\right)}{\left(E_{i}\left(\boldsymbol{k}_{\|, i}\right)-E_{m}\left(\boldsymbol{k}_{\|, f}\right) \mp \hbar \omega_{\mathrm{ph}}\right)^{2}} d(\hbar \bar{\omega}),
\end{aligned}
$$

where $G(\hbar \omega)$ is the density of states of the electromagnetic field, $F_{\text {ind }}$ is a proportionality constant, and $E_{i}, E_{m}$, and $E_{f}$ are the energy of the carriers in the $\Delta, \Gamma_{c}$, and $\Gamma_{v}$ bands, respectively. To evaluate the above expression, we performed a double integral over the excess energy of the $\Delta_{c}$ and $\Gamma_{v}$ carriers. We calculated the polarization-dependent dipole matrix elements $\boldsymbol{p}_{c v}^{\Gamma}$ at the $\Gamma$ point by means of a $s p^{3} d^{5} s^{*}$ first-neighbor tight binding code [31] and $n_{\mathrm{ph}}$ using Bose-Einstein statistics.

The relevant material parameters used to describe the strained SiGe QW alloy were obtained by linear interpolation of the corresponding $\mathrm{Si}$ and Ge values, which have been taken from the literature (see Table III in Ref. [17] and Table I in Ref. [31]). The offset at the interface for the energy of the barycenter of the valence $\mathrm{HH}, \mathrm{LH}$, and SO bands has been calculated according to Ref. [32].

\section{RESULTS AND DISCUSSION}

\section{A. Structural analysis}

As a consequence of the $4.2 \%$ lattice mismatch between $\mathrm{Ge}$ and $\mathrm{Si}$, pure $\mathrm{Ge}$ grown on a $\mathrm{Si}$ substrate experiences compressive strain. In equilibrium conditions, this strain is released via the spontaneous formation of Ge dots, on top of a coherent Ge wetting layer, following the so-called StranskiKrastanow growth mode. The critical thickness of the wetting layer at which dot formation occurs is a function of growth temperature and can exceed several monolayers [33]. As can be seen from bright-field transmission electron microscopy (BF-TEM) [Fig. 2(a)] and HR-TEM [Fig. 2(b)] images, because of the low growth temperature chosen here, the Ge layer thickness was below the critical thickness necessary for island formation, and the resulting sample contains $\mathrm{SiGe}$ QWs rather than dots [34]. The sample is crystalline and no threading dislocations are visible, indicating that the growth strategy employed enabled us to produce a sequence of well-defined QW structures. Moreover, the low deposition temperature used allows a reduction of the $\mathrm{Si}-\mathrm{Ge}$ intermixing [35]. The HR-TEM images allow us to estimate the thickness of the Ge-rich regions to be in the 1.1 to $1.4 \mathrm{~nm}$ range.

To obtain more information on the composition and lattice strain of the QWs, we used Raman and XRD analysis. Raman data of our sample and that of a bulk Si (001) reference are shown in Fig. 2(c).

A number of investigations have been devoted to obtaining models for the extraction of composition and strain data from Raman analysis of SiGe island structures or thin pseudomorphic films [36-40]. To isolate the signal coming from the multiple QWs, we follow Refs. [37,38] and subtract the Si spectrum $I_{\mathrm{Si}}$ from the sample spectrum $I_{\mathrm{S}}$, i.e., $I_{\mathrm{S}}-F I_{\mathrm{Si}}$, where $F$ is a scaling factor obtained by taking the ratios of the Si peaks at $520 \mathrm{~cm}^{-1}$. From the resulting multiple-QW spectrum, we obtain for the $\mathrm{Ge}-\mathrm{Ge}$ and $\mathrm{Ge}-\mathrm{Si}$ modes the frequencies $\omega_{\mathrm{Ge}-\mathrm{Ge}}=$ $303.95 \mathrm{~cm}^{-1}$ and $\omega_{\mathrm{Si}-\mathrm{Ge}}=421.27 \mathrm{~cm}^{-1}$, respectively.

From these values, we can estimate the Ge content, $x$, and the biaxial strain, $\varepsilon_{x x}$, in the well region using empirical relationships [38]:

$$
\begin{aligned}
\omega_{\mathrm{Si}-\mathrm{Ge}} & =400+29 x-95 x^{2}+213 x^{3}-170 x^{4}+b_{\mathrm{Si}-\mathrm{Ge}} \varepsilon_{x x}, \\
\omega_{\mathrm{Ge}-\mathrm{Ge}} & =283+5 x+12 x^{2}+b_{\mathrm{Ge}-\mathrm{Ge}} \varepsilon_{x x} .
\end{aligned}
$$

For the strain-shift coefficients $b_{\mathrm{Ge}-\mathrm{Ge}}$ and $b_{\mathrm{Si}-\mathrm{Ge}}$, we used the empirical relations given in Ref. [41], determined 

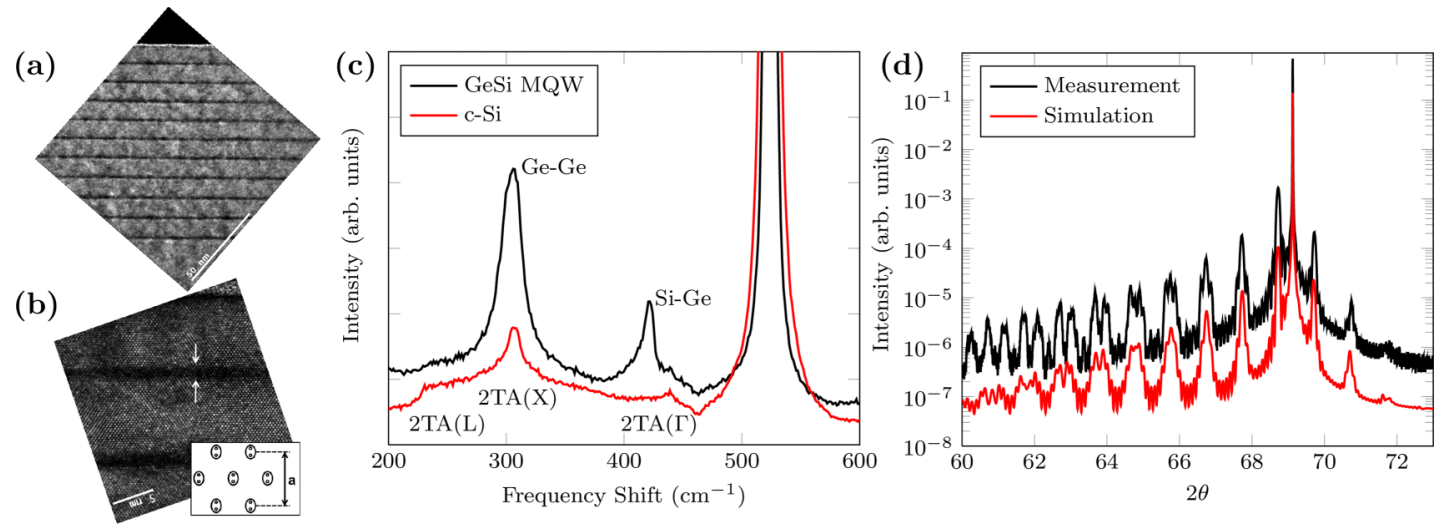

FIG. 2. (a) BF-TEM and (b) HR-TEM cross-section images of the Ge multiple-QW structure. (c) Overlay of c-Si (red) and Ge multiple-QW sample (black) Raman signal. (d) XRD $\omega-2 \theta$ scan along the (004) direction of the Ge multiple-QW sample (black) and fit with a multiple square well model (red).

experimentally for pseudomorphic $\mathrm{Si}_{1-x} \mathrm{Ge}_{x}$ layers as a function of Ge content $x$ :

$$
\begin{aligned}
b_{\mathrm{Si}-\mathrm{Ge}} & =-190(15)(x-1)^{4}-555(15) \mathrm{cm}^{-1}, \\
b_{\mathrm{Ge}-\mathrm{Ge}} & =-190(15)(x-1)^{4}-460(20) \mathrm{cm}^{-1} .
\end{aligned}
$$

We obtained an average Ge content of $x=(0.68 \pm 0.01)$ within the QW layer and a compressive strain of $\varepsilon_{x x}=$ $-2.64 \%$. The main errors in this analysis originate not only from the uncertainties associated with the strain-shift coefficients but also from the fact that both Ge content and strain could be inhomogeneously distributed within the QWs.

Further insights into the multiple-QW structures were obtained by XRD measurements. Figure 2(d) shows the results of an $\omega-2 \theta$ scan along the (004) direction, together with diffraction simulation results. The large number of higher order superlattice peaks indicates good homogeneity of the QWs within the sample. To simulate the angle-dependent intensity, sharp interfaces were assumed for the Ge-rich QWs whose geometrical parameters (well width and Ge content) were used for data fitting. Good agreement between experiment and simulation was obtained for an average $\mathrm{Ge}$ content of $x_{\mathrm{av}}=0.63$ and an average well thickness of $w_{\mathrm{av}}=(1.2 \pm 0.2) \mathrm{nm}$. Assuming pseudomorphic strain but neglecting material diffusion (i.e., assuming a box profile of the Ge content within the well), this analysis yields a total of 4.9 ML of Ge deposited, which is close to the nominal value of 5.5 ML used in the growth process.

Summing up all the information acquired from HR-TEM, Raman spectroscopy, and XRD, we can conclude that the growth method applied produces well-defined periodic QW structures with average Ge content in the wells between $63 \%$ and $68 \%$ and thicknesses in the range of $1.2-1.4 \mathrm{~nm}$.

\section{B. Optical properties}

The samples' optical properties have been investigated by $\mu \mathrm{PL}$ at different pump power densities and sample temperatures. We first discuss measurements performed at a constant lattice temperature of $T_{L}=80 \mathrm{~K}$ and varying the pump power densities [Fig. 3(a)]. The PL intensity increases by a factor of 20 when the excitation density increases from $3.2 \times 10^{4}$ to
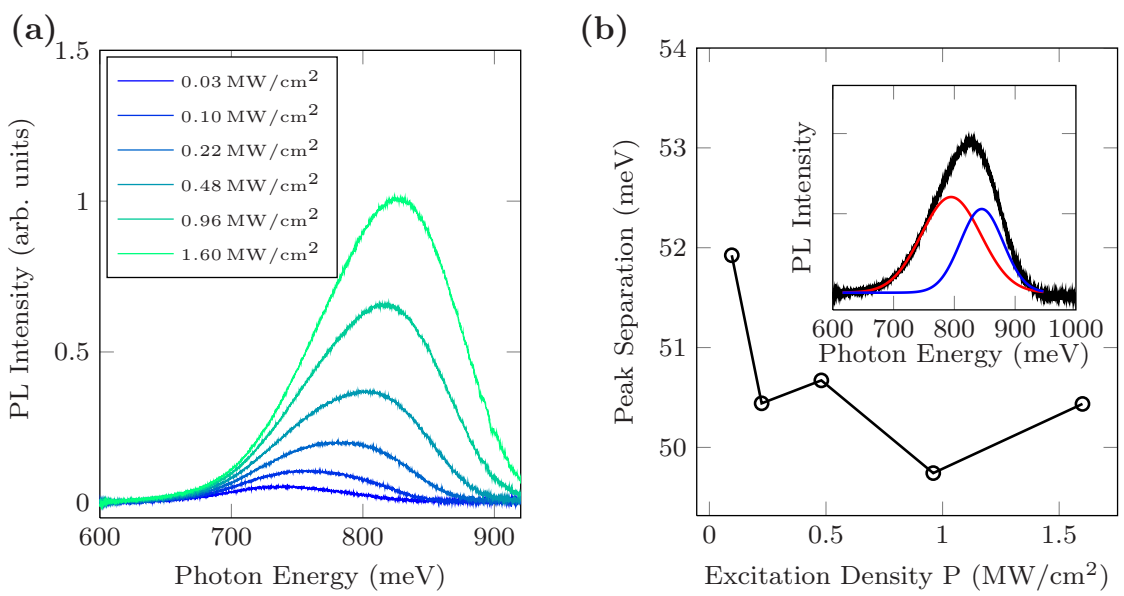

(c)

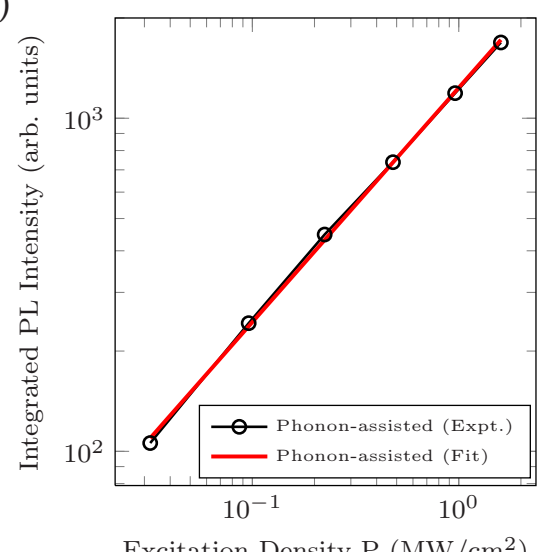

FIG. 3. (a) Experimentally observed PL spectra at $T_{L}=80 \mathrm{~K}$ for different excitation densities. (b) Measured energy separation between phonon-assisted and NP peaks as a function of excitation density; in the inset, the deconvolution of the PL signal taken at $80 \mathrm{~K}^{\text {and }} 1.60 \mathrm{MWcm}^{-2}$ with two Gaussian peaks is shown. (c) Experimentally measured integrated PL intensity for the phonon-assisted peak as a function of the excitation power density. 
$160 \times 10^{4} \mathrm{Wcm}^{-2}$ and blueshifts from 740 to $830 \mathrm{meV}$. As shown in the inset of Fig. 3(b), two distinct features contribute to the PL signal. Gaussian deconvolution analysis indicates that their energy separation is $\sim 51 \mathrm{meV}$ over the entire pump power range. As we suspect that electrons are localized mainly within the barrier region due to the type II confinement, we attribute this energy difference to the TO phonon energy of bulk $\mathrm{Si}$ [30]. However, as already mentioned above, the Si-Ge vibrational mode at the QW interface, whose energy is close to the TO optical phonon of the Si lattice, may contribute to the lower energy spectral peak. While the lower energy feature can be attributed to an indirect band-to-band recombination assisted by spontaneous emission of phonons with appropriate momentum, the higher energy must be related to the presence of an elastic scattering channel (NP), which provides the missing momentum for the same indirect transition. The observed blueshift can be attributed to pump-induced band bending, which affects the confinement energy in the type II band alignment of the investigated multiple QWs. In type II heterostructures, the blueshift of the PL peak energy as a function of pump power follows a power-law dependence of the form $\Delta E_{\text {peak }} \propto P^{r}$. After a detailed analysis of the spectra in Fig. 3(a), we determined a scaling exponent $r=0.2$, in close agreement with values reported in the literature for similar type II structures [42]. Moreover, the integrated PL intensity $I$, as a function of pump power, can be described by a power law $I \propto P^{m}$ [40]. From Fig. 3(c), where the integrated PL signal related to the phonon-assisted feature is shown as a function of the excitation density, we measure a scaling exponent value $m=0.70 \pm 0.01$, close to the value of $2 / 3$, obtained when the recombination dynamics is dominated by the Auger mechanism. In this case, the generation rate, which is proportional to the pump intensity, scales as the third power of the excess carrier density $\delta n$, which can be obtained by considering that in steady-state conditions it must be equal to the Auger recombination rate. It follows that for intrinsic samples $I \propto P^{2 / 3}$, since the integrated PL signal is approximately proportional to $\delta n^{2}$.

As a next step, we use our theoretical model to gain a deeper understanding of the physical processes causing the observed energy shift and spectral broadening. To this aim, the composition profile of the sample has been modeled as a square profile with a constant Ge concentration of $68 \%$ within the QW, which corresponds to the average Ge concentration measured by Raman spectroscopy. The assumption of a square well is only an approximation of the Ge distribution within the sample, which is likely to be influenced by segregation effects. However, determining the position-dependent Ge distribution within the QWs would necessitate an experimental technique with a subnanometer resolution. In our ultrathin $\mathrm{SiGe} / \mathrm{Si}$ multiple QWs, the calculated transition energies are quite sensitive to small variations of the well thickness, due to the relevant role played by the confinement in the valence band. As a consequence, in our simulations we tuned, starting from the HR-TEM and XRD measurements, the QW thickness of the adopted square profile to improve the agreement between theoretical and experimental PL peak energies. An effective QW thickness of $1.6 \mathrm{~nm}$ is only slightly larger than the XRD estimates of $(1.2 \pm 0.2) \mathrm{nm}$ and places our theoretical results within $20 \mathrm{meV}$ of the experimentally determined values. The remaining difference has the order of magnitude of the exciton binding energy, which is not accounted for in our theoretical model. To further justify the introduction of this effective square well thickness, different material parameters, such as band offsets and confinement masses, whose precise values are unknown, influence the calculated transition energies. For instance, increasing the $\mathrm{HH}$ mass along the confinement direction by $10 \%$ results in an increase of the transition energy by about $10 \mathrm{meV}$. An effective QW thickness for the multiple-QW square profile can then be regarded, as often proposed in the literature, as a way to account for uncertainties associated with the relevant material parameters.

Furthermore, the optically excited carrier density $\delta n$ within the QW region depends on the defect density and all other material parameters controlling the nonradiative recombination rate, which are largely unknown for our sample. Therefore, to estimate $\delta n$, we rely on a phenomenological relation

$$
\delta n=C P^{0.37},
$$

where $C$ is a fitting constant. The exponent value of 0.37 has been chosen to reproduce the scaling exponent measured for the integrated TO signal $m=(0.70 \pm 0.01)$, and as one can expect, it is found to be approximately equal to $m / 2$. The fitting parameter $C$ has been fixed to reproduce the measured pump-induced energy blueshift. As discussed in the following, the blueshift is related to band bending effects, which is due to electron and hole spatial separation and whose magnitude is controlled by the amount of the induced excess carrier density. This calibration procedure returns $C=3.5 \times 10^{11} \mathrm{~W}^{-0.75} \mathrm{~cm}^{0.5}$, giving an indication that at $T_{L}=80 \mathrm{~K}$, the excess carrier density range is $1.2 \times 10^{12}$ to $5.0 \times 10^{12} \mathrm{~cm}^{-2}$ when the pump power density is varied from $3.2 \times 10^{4}$ to $160 \times 10^{4} \mathrm{Wcm}^{-2}$. Numerical values for NP and phonon-assisted peak energy as a function of the pump power are compared with the experimental data in Fig. 4(a). The NP curve has been obtained by upshifting numerical data for the phonon-assisted feature by $58.8 \mathrm{meV}$. Because of the large phonon energy of $\mathrm{Si}$ with respect to $k T_{L}$, the phonon-assisted signal is related to spontaneous phonon emission only.

To elucidate the cause of the observed blueshift, which is a typical signature of type II band alignment [43], we compare the band edge electronic states at low and high excitation densities as shown in Fig. 5. Due to the spatial separation of the excess hole and electrons, which are mainly localized in the $\mathrm{SiGe}$ and $\mathrm{Si}$ regions, respectively, a larger pump power density increases the band bending. The HH1 energy is quite insensitive to this effect due to the large values of both the offset and the confinement energy in the valence band. In contrast, the nondegenerate $\Delta_{2}$ and $\Delta_{4}$ subband states are more sensitive to the band edge profile, because they are localized in the thicker $\mathrm{Si}$ region. It follows that when the pump power increases, the conduction confinement energy becomes larger and, as a result, the PL peak energy blueshifts. A comparison of experimental and simulated PL spectra originating from the phonon-assisted recombination evaluated at $T_{L}=80 \mathrm{~K}$ for different pump powers is shown in Fig. 4(b). Although the experimental spectra are slightly broader, probably due to fluctuations in material composition and QW width, we find that the pump-induced increase of the 

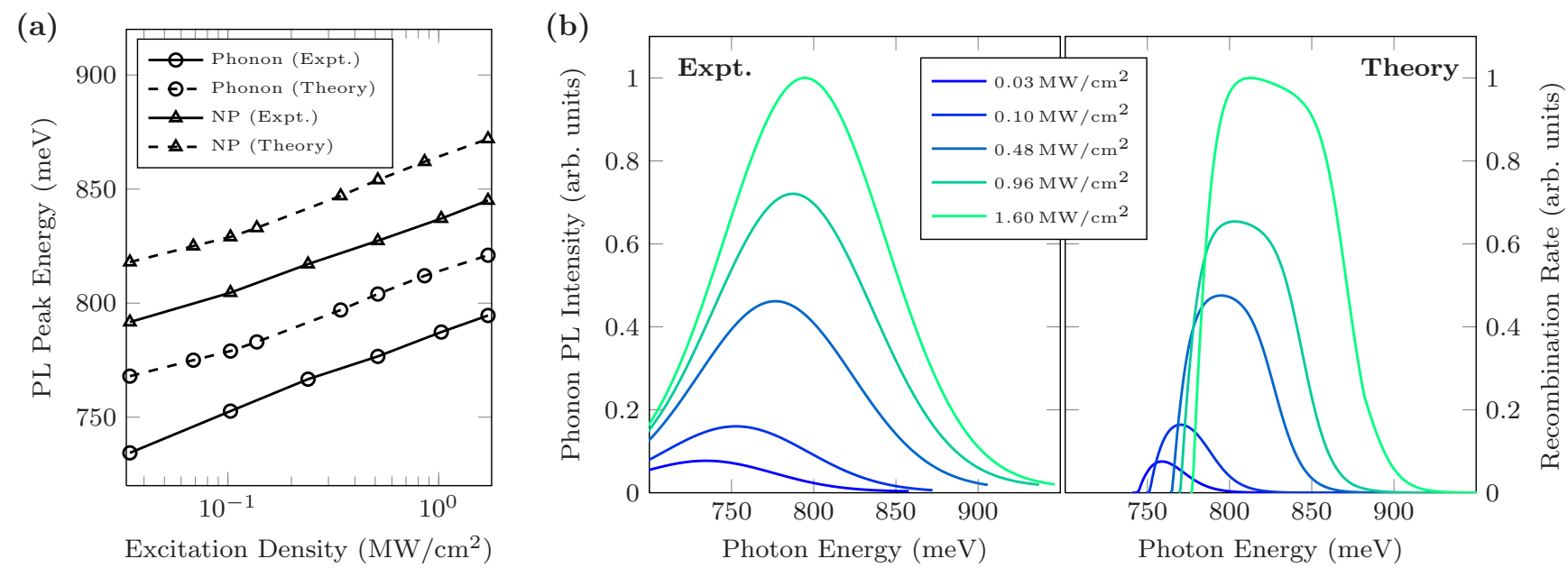

FIG. 4. (a) PL peak energy of the phonon-assisted and NP line according to theory and experiment as a function of the excitation density. (b) Comparison of experimental and simulated phonon-assisted PL at $T_{L}=80 \mathrm{~K}$ for different pump powers.

peak intensity is well reproduced by the model. We stress here that this is not a priori obvious, because the phenomenological relation between excess carrier and pump density was tuned to reproduce the energy shift, not the PL peak intensity.

PL spectra have been also studied at a constant pump power density of $9.6 \times 10^{4} \mathrm{Wcm}^{-2}$ with varying $T_{L}$ in the 80-300 K range [Fig. 6(a)]. With increasing temperature, the spectra become broader and the PL peak energy blueshifts by $\sim 30 \mathrm{meV}$. The PL intensity is not significantly quenched up to room temperature, the total integrated intensity being reduced by a factor of 3 only when $T_{L}$ is increased from 80 to $300 \mathrm{~K}$. Regarding the spectral blueshift, this behavior seems, at first, to be in conflict with the temperature-driven shrinking of both the $\mathrm{Si}$ and the Ge band gaps. To shed light on this unusual trend, we again resolve the phonon-assisted and NP feature by Gaussian deconvolution. Due to thermal broadening effects,

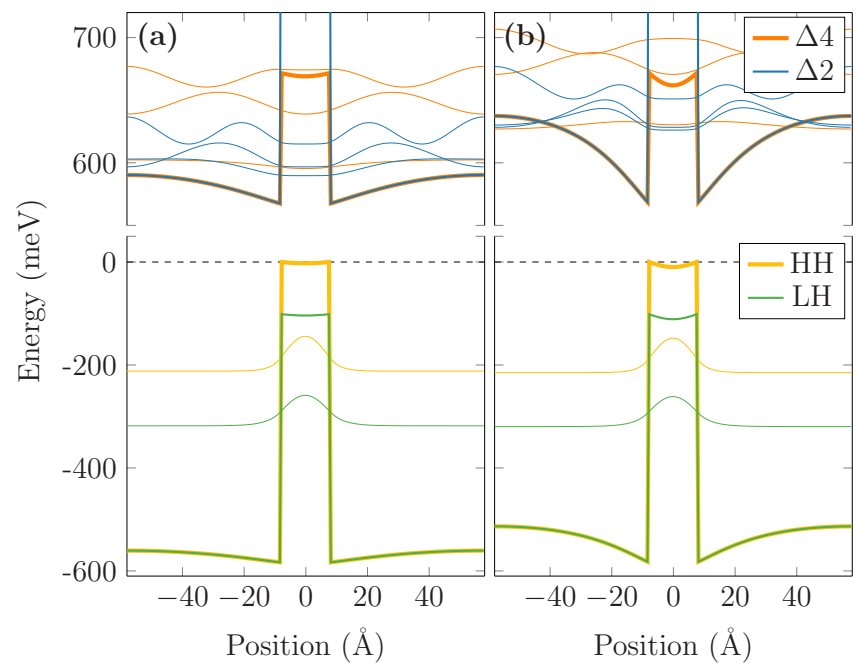

FIG. 5. Near-gap subband states calculated for $T_{L}=80 \mathrm{~K}$ at low (a) and high (b) optical excitation densities. Confinement energies in the valence band are unchanged, while at high excitation density, the conduction subband states are found at higher energies due to larger band bending. this kind of analysis was significant only for $T_{L}<200 \mathrm{~K}$. We find that when looking at spectrally resolved features, the expected temperature dependence is observed, because both the NP and the phonon-assisted energy peaks decrease monotonically by roughly $30 \mathrm{meV}$, as shown in Fig. 7(a). To further explain this finding, we theoretically investigated the PL spectra as a function of temperature. To this aim, the phonon-assisted contribution of the PL spectra for the $9.6 \times 10^{4} \mathrm{Wcm}^{-2}$ excitation density at a given $T_{L}$ has been calculated by tuning the excess carrier density to reproduce the measured ratio between the integrated phonon-assisted signal at temperature $T_{L}$ and that at $T_{L}{ }^{\prime}=80 \mathrm{~K}$, for which we already know the excess carrier density from the investigation of the excitation density measurements. From this calibration procedure, we estimate an excess carrier density decrease for the 80-200 K temperature variation by a factor of 0.3 only. Numerical phonon-assisted and NP peak energies as a function of temperature are compared with the experimental counterpart in Fig. 7(a). It is apparent from Fig. 7(a) that apart from the already-mentioned overestimation of about $20 \mathrm{meV}$, the numerical data fully capture the observed trend.

The Gaussian fits of the phonon-assisted PL feature at different temperatures are compared with numerical data in Fig. 6(b). Although the experimental spectra are broader, presumably due to multiple-QW thickness fluctuation in our samples, it is remarkable that the ratios among different peak intensities are in good agreement with experiment. This was not obvious a priori, because in the calibration procedure, the integrated intensities were targeted. This indicates that the thermal contributions to the broadening are correctly reproduced by the model. From the above discussion, we conclude that the phonon-assisted PL peak energy redshift is dominated by the temperature-dependent decrease of the band gap, while the thermal excitation of higher energy states, which in principle could drive a blueshift, plays only a minor role. The observed blueshift in the total PL spectrum is then to be attributed to a spectral weight shift from the phonon-assisted feature, dominating at low temperature, to the higher energy NP contribution, which becomes dominant at higher $T_{L}$. This effect more than compensates for the 

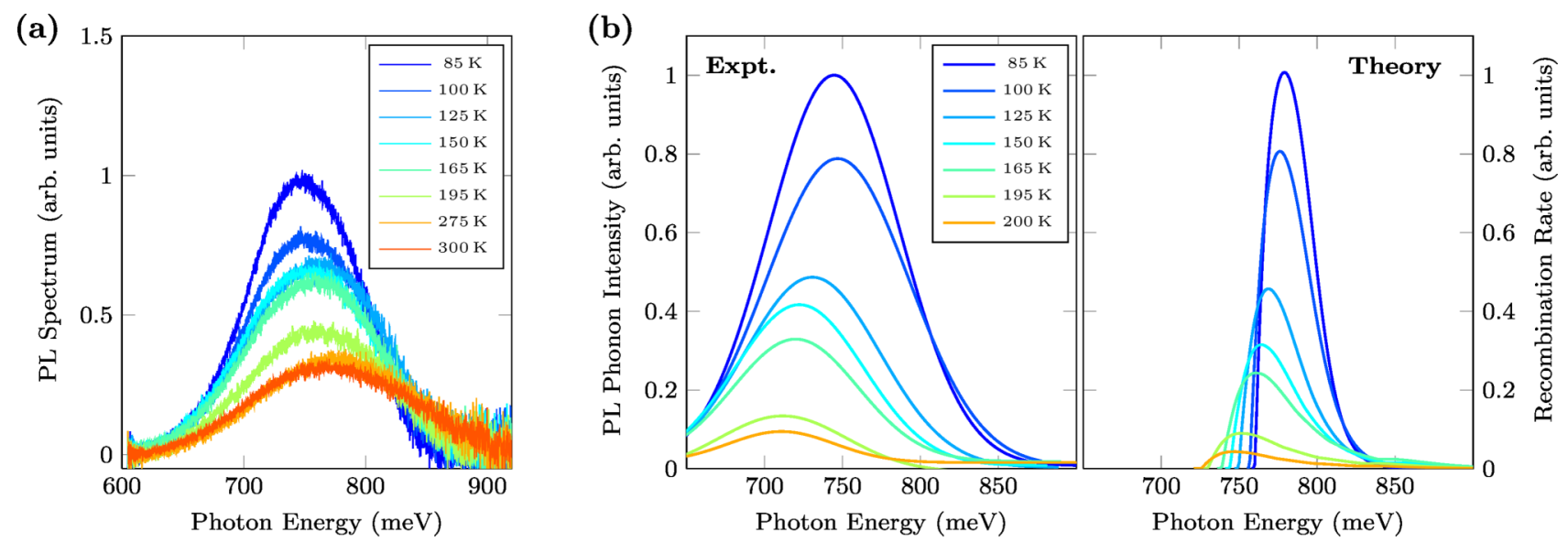

FIG. 6. (a) Experimental PL spectra at a $0.10 \mathrm{Wcm}^{-2}$ excitation density for different lattice temperatures. (b) Comparison between phonon-assisted peaks extracted from experiment and theory for different lattice temperatures.

band gap shrinkage. Considering the $30 \mathrm{meV}$ redshift of the phonon-assisted and NP peaks predicted by our model and that their energy is separated by about $58.8 \mathrm{meV}$, we estimate a blueshift of the total PL spectrum of about $30 \mathrm{meV}$, which well matches the measured value of $20 \mathrm{meV}$ between 80 and $200 \mathrm{~K}$. To further consolidate this interpretation, we observe that the measured ratio between the phonon-assisted and the NP-integrated PL signals, $\sim 10$ at $T_{L}=80 \mathrm{~K}$, decreases to $\sim 0.15$ at $T_{L}=200 \mathrm{~K}$ [Fig. 7(b)]. The thermal quenching of the PL is therefore hindered by a strong increase of the NP intensity at higher $T_{L}$. This behavior can be explained by looking into the temperature dependence of the phononassisted and NP features separately. For the phonon-assisted feature, the coupling between electrons and phonons can be approximately described as temperature independent over the investigated temperature range due to the large phonon energy with respect to $\mathrm{k} T_{L}$ (spontaneous phonon emission). It follows that the phonon-assisted signal intensity is mainly governed by the decreasing excess electron density, related to a faster nondegenerate recombination dynamics with increasing temperature. However, from Fig. 7(b), we infer that the electron-hole coupling of the NP recombination must strongly increase with $T_{L}$ to overcompensate for the lower excess carrier density. We speculate that two mechanisms could be responsible for this behavior.

At higher temperatures, holes and electrons occupy states with higher in-plane momenta. These larger momenta increase the rate at which charge carriers encounter interface defects. Since these elastic scattering events can provide the missing momentum for indirect transitions, an increased NP recombination rate is to be expected. An alternative explanation for the $T_{L}$-driven increase of the NP electron-hole coupling is that at higher temperature conduction electrons populate higher excited subbands whose wave function penetrates deeper into the $\mathrm{SiGe}$ region (Fig. 5). This may enhance the interface roughness scattering rate contributing to the NP PL feature. In this case, the enhanced contribution from excited conduction subbands will slightly blueshift the NP feature, resulting in an (a)

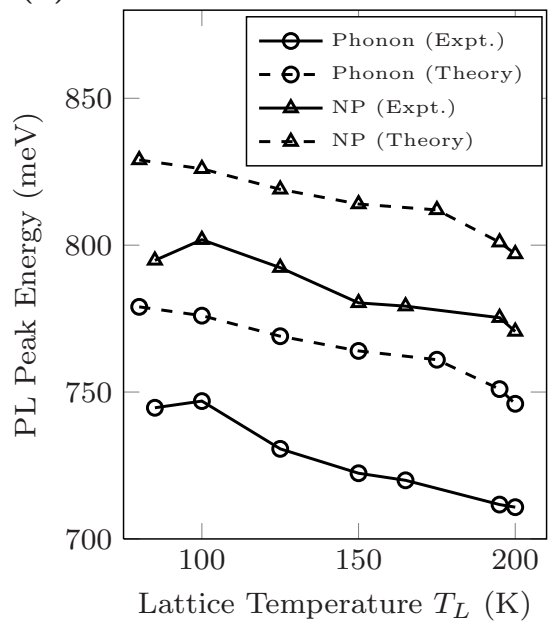

(b)

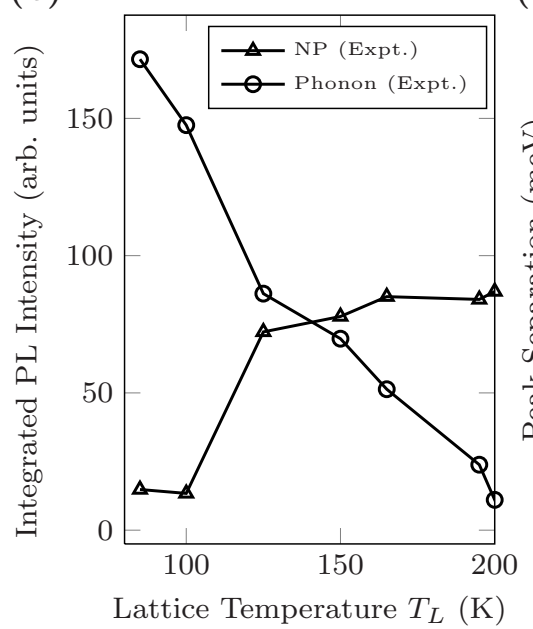

(c)

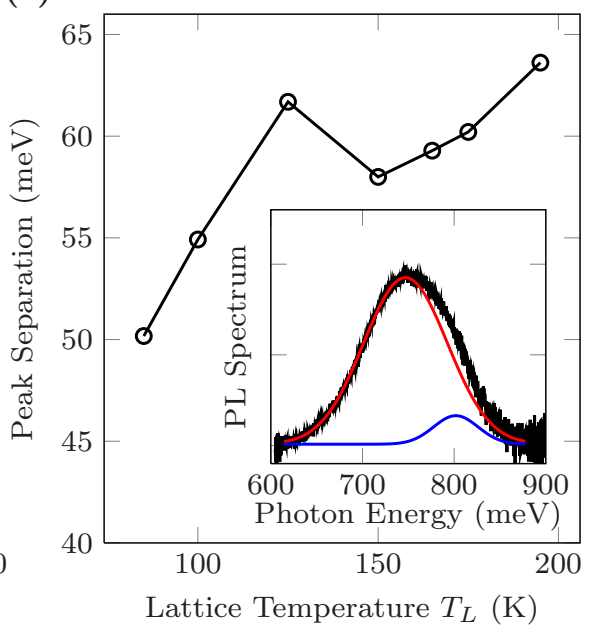

FIG. 7. (a) PL peak energies as a function of $T_{L}$ for theory and experiment. (b) Experimental integrated PL signal as a function of $T_{L}$. (c) Measured energy separation of phonon-assisted and NP peaks as a function of $T_{L}$; in the inset, the deconvolution of the PL signal taken at $100 \mathrm{~K}$ and $0.1 \mathrm{MW} \mathrm{cm}^{-2}$ with two Gaussian peaks is shown. 
increase in energy separation with the phonon-assisted peak. This effect could explain the trend shown in Fig. 7(c).

\section{CONCLUSIONS}

The two main challenges in using SiGe-based QW structures for optoelectronic applications are the usually strong thermal quenching of their luminescence and the complexity of manufacturing SiGe QWs with high Ge concentrations directly on a $\mathrm{Si}$ substrate. The growth strategy presented here appears to circumvent both challenges. By employing low growth temperatures and limiting the deposition of Ge to less than 5.5 ML, a multiple-QW structure consisting of $\mathrm{Si}_{1-x} \mathrm{Ge}_{x}$ layers with a Ge concentration exceeding $60 \%$ can be directly grown on a Si substrate. Furthermore, we find that the PL of this structure is unusually robust against thermal quenching. Employing an empirical relationship between the optically excited charge carrier density and the excitation density of the PL setup and using a self-consistent effective mass approach, we were able to fully reproduce the phonon-related features of the PL spectra. We find that while the phonon-related PL intensity decreases when the temperature is increased, due to the increase of the nondegenerate recombination rate and as a result of the reduced charge carrier density, the NP-related PL intensity increases. This increase of the NP intensity more than compensates for the decrease in intensity related to the phonon-assisted transitions and leads to a shift in spectral weight from the phonon-assisted to the NP feature, effectively blueshifting the total PL energy. We conjecture that the increase in the NP-related recombination rate is due to the higher in-plane momenta of the charge carriers at higher temperatures, which increases the rate at which charge carriers encounter elastic scattering centers. Alternatively, this effect might be related to an enhanced interface scattering rate as a result of the occupation of higher energy levels by the optically excited electrons. The wave functions related to those higher energy levels penetrate deeper into the interface region between the $\mathrm{Si}$ and the $\mathrm{SiGe}$ regions, enhancing the contribution of the interface roughness scattering rate to the NP signal.

From the current state of our investigation, we deem that the mechanism limiting the effect of thermal quenching is associated with the type II confinement typical for SiGe/Si QWs. It would be interesting to investigate similar layer structures in other material systems that exhibit type II confinement.

\section{ACKNOWLEDGMENTS}

T.W. and K.B. were supported by the Stiftung der Deutschen Wirtschaft (sdw) and by the Deutsche Forschungsgemeinschaft (DFG) through project B10 within the Collaborative Research Center (CRC) 951 Hybrid Inorganic/Organic Systems for Opto-Electronics. HRTEM measurements were financed by Xunta de Galica Grant No. GRC2014/008.
[1] F. Schäffler, High-mobility Si and Ge structures, Semicond. Sci. Tech. 12, 1515 (1997).

[2] K. L. Wang, J. L. Liu, and G. Jin, Self-assembled Ge quantum dots on Si and their applications, J. Cryst. Growth 237, 1892 (2002).

[3] R. A. Soref, Silicon-based group IV heterostructures for optoelectronic applications, J. Vac. Sci. Tech. 14, 913 (1996).

[4] L. Tsybeskov, E.-K. Lee, H.-Y. Chang, D. J. Lockwood, J.-M. Baribeau, X. Wu, and T. I. Kamins, Silicon-germanium nanostructures for on-chip optical interconnects, Appl. Phys. Mater. Sci. Process. 95, 1015 (2009).

[5] M. Wachter, F. Schäffler, H.-J. Herzog, K. Thonke, and R. Sauer, Photoluminescence of high-quality SiGe quantum wells grown by molecular beam epitaxy, Appl. Phys. Lett. 63, 376 (1993).

[6] H. Sunamura, Y. Shiraki, and S. Fukatsu, Growth mode transition and photoluminescence properties of $\mathrm{Si}_{1-x} \mathrm{Ge}_{x} / \mathrm{Si}$ quantum well structures with high Ge composition, Appl. Phys. Lett. 66, 953 (1995).

[7] Y. Shiraki, H. Sunamura, N. Usami, and S. Fukatsu, Formation and optical properties of SiGe/Si quantum structures, Appl. Surf. Sci. 102, 263 (1996).

[8] N. Pinto, F. Tombolini, R. Murri, M. De Crescenzi, M. Casalboni, G. Barucca, and G. Majni, Photoluminescence investigation of short period silicon-germanium heterostructures grown using molecular beam epitaxy, Surf. Sci. 437, 145 (1999).

[9] J.-P. Noël, N. L. Rowell, D. C. Houghton, and D. D. Perovic, Intense photoluminescence between 1.3 and $1.8 \mu \mathrm{m}$ from strained $\mathrm{Si}_{1-x} \mathrm{Ge}_{x}$ alloys, Appl. Phys. Lett. 57, 1037 (1990).

[10] J. C. Sturm, H. Manoharan, L. C. Lenchyshyn, M. L. W. Thewalt, N. L. Rowell, J.-P. Noël, and D. C. Houghton, Well-
Resolved Band-Edge Photoluminescence of Excitons Confined in Strained $\mathrm{Si}_{1-x} \mathrm{Ge}_{x}$ Quantum Wells, Phys. Rev. Lett. 66, 1362 (1991).

[11] Y.-H. Kuo, Y. K. Lee, Y. Ge, S. Ren, J. E. Roth, T. I. Kamins, D. A. B. Miller, and J. S. Harris, Strong quantum-confined Stark effect in germanium quantum-well structures on silicon, Nature 437, 1334 (2005).

[12] Y.-H. Kuo, Y. K. Lee, Y. Ge, S. Ren, J. E. Roth, T. I. Kamins, D. A. B. Miller, and J. S. Harris, Quantum-confined Stark effect in $\mathrm{Ge} / \mathrm{SiGe}$ quantum wells on Si for optical modulators, IEEE J. Sel. Top. Quant. Electron. 12, 1503 (2006).

[13] E. Gatti, E. Grilli, M. Guzzi, D. Chrastina, G. Isella, and H. Von Känel, Room temperature photoluminescence of Ge multiple quantum wells with Ge-rich barriers, Appl. Phys. Lett. 98, 031106 (2011).

[14] R. P. G. Karunasiri, J. S. Park, Y. J. Mii, and K. L. Wang, Intersubband absorption in $\mathrm{Si}_{1-x} \mathrm{Ge}_{x} / \mathrm{Si}$ multiple quantum wells, Appl. Phys. Lett. 57, 2585 (1990).

[15] K. Gallacher, A. Ballabio, R. W. Millar, J. Frigerio, A. Bashir, I. MacLaren, G. Isella, M. Ortolani, and D. J. Paul, Mid-infrared intersubband absorption from p-Ge quantum wells grown on $\mathrm{Si}$ substrates, Appl. Phys. Lett. 108, 091114 (2016).

[16] M. De Seta, G. Capellini, M. Ortolani, M. Virgilio, G. Grosso, G. Nicotra, and P. Zaumseil, Narrow intersubband transitions in n-type Ge/SiGe multi-quantum wells: Control of the terahertz absorption energy trough the temperature dependent depolarization shift, Nanotechnology 23, 465708 (2012).

[17] Y. Busby, M. De Seta, G. Capellini, F. Evangelisti, M. Ortolani, M. Virgilio, G. Grosso, G. Pizzi, P. Calvani, S. Lupi, M. Nardone, G. Nicotra, and C. Spinella, Near-and far-infrared absorption and 
electronic structure of Ge-SiGe multiple quantum wells, Phys. Rev. B 82, 205317 (2010).

[18] R. Bates, S. A. Lynch, D. J. Paul, Z. Ikonic, R. W. Kelsall, P. Harrison, S. L. Liew, D. J. Norris, A. G. Cullis, W. R. Tribe, and D. D. Arnone, Interwell intersubband electroluminescence from $\mathrm{Si} / \mathrm{SiGe}$ quantum cascade emitters, Appl. Phys. Lett. 83, 4092 (2003).

[19] I. A. Buyanova, W. M. Chen, G. Pozina, B. Monemar, W.-X. $\mathrm{Ni}$, and G. V. Hansson, Mechanism for thermal quenching of luminescence in $\mathrm{SiGe} / \mathrm{Si}$ structures grown by molecular beam epitaxy: Role of nonradiative defects, Appl. Phys. Lett. 71, 3676 (1997).

[20] D. J. Lockwood, X. Wu, J.-M. Baribeau, S. A. Mala, X. Wang, and L. Tsybeskov, Si/SiGe heterointerfaces in one-, two-, and three-dimensional nanostructures: Their impact on SiGe light emission, Front. Mater. 3, 12 (2016).

[21] Y. Shiraki and A. Sakai, Fabrication technology of SiGe heterostructures and their properties, Surf. Sci. Rep. 59, 153 (2005).

[22] T. Baier, U. Mantz, K. Thonke, R. Sauer, F. Schäffler, and H.-J. Herzog, Type-II band alignment in $\mathrm{Si} / \mathrm{Si}_{1-x} \mathrm{Ge}_{x}$ quantum wells from photoluminescence line shifts due to optically induced band-bending effects: Experiment and theory, Phys. Rev. B 50, 15191 (1994).

[23] M. Virgilio, R. Farchioni, and G. Grosso, Theoretical investigation of near gap electronic states of $\mathrm{Si} / \mathrm{SiGe}$ multiple quantum wells on (001)-Si or SiGe substrates, J. Appl. Phys. 99, 053710 (2006).

[24] P. K. Basu and S. K. Paul, Ideal interband absorption spectra in an indirect-gap-semiconductor quantum well, Phys. Rev. B 46, 13389 (1992).

[25] H. H. Hassan and H. N. Spector, Optical absorption in semiconducting quantum-well structures: Indirect interband transitions, Phys. Rev. B 33, 5456 (1986).

[26] C. G. Van de Walle, Band lineups and deformation potentials in the model-solid theory, Phys. Rev. B 39, 1871 (1989).

[27] C. Y.-P. Chao and S. L. Chuang, Spin-orbit-coupling effects on the valence-band structure of strained semiconductor quantum wells, Phys. Rev. B 46, 4110 (1992).

[28] M. V. Fischetti, Monte Carlo simulation of transport in technologically significant semiconductors of the diamond and zinc-blende structures. I. Homogeneous transport, IEEE Trans. Electron. Dev. 38, 634 (1991).

[29] C. Jacoboni, F. Nava, C. Canali, and G. Ottaviani, Electron drift velocity and diffusivity in germanium, Phys. Rev. B 24, 1014 (1981).

[30] J. Weber and M. I. Alonso, Near-band-gap photoluminescence of Si-Ge alloys, Phys. Rev. B 40, 5683 (1989).
[31] M. Virgilio, C. L. Manganelli, G. Grosso, G. Pizzi, and G. Capellini, Radiative recombination and optical gain spectra in biaxially strained n-type germanium, Phys. Rev. B 87, 235313 (2013).

[32] C. G. Van de Walle and R. M. Martin, Theoretical calculations of heterojunction discontinuities in the $\mathrm{Si} / \mathrm{Ge}$ system, Phys. Rev. B 34, 5621 (1986).

[33] R. Bergamaschini, M. Brehm, M. Grydlik, T. Fromherz, G. Bauer, and F. Montalenti, Temperature-dependent evolution of the wetting layer thickness during Ge deposition on Si (001), Nanotechnology 22, 285704 (2011).

[34] F. Oliveira, I. A. Fischer, A. Benedetti, M. F. Cerqueira, M. I. Vasilevskiy, S. Stefanov, S. Chiussi, and J. Schulze, Multi-stacks of epitaxial GeSn self-assembled dots in Si: Structural analysis, J. Appl. Phys. 117, 125706 (2015).

[35] G. Capellini, M. De Seta, and F. Evangelisti, SiGe intermixing in Ge/Si (100) islands, Appl. Phys. Lett. 78, 303 (2001).

[36] J. C. Tsang, P. M. Mooney, F. Dacol, and J. O. Chu, Measurements of alloy composition and strain in thin $\mathrm{Ge}_{x} \mathrm{Si}_{1-x}$ layers, J. Appl. Phys. 75, 8098 (1994).

[37] P. H. Tan, K. Brunner, D. Bougeard, and G. Abstreiter, Raman characterization of strain and composition in small-sized selfassembled Si/Ge dots, Phys. Rev. B 68, 125302 (2003).

[38] M. I. Alonso, M. De la Calle, J. O. Osso, M. Garriga, and A. R. Goni, Strain and composition profiles of self-assembled $\mathrm{Ge} / \mathrm{Si}$ (001) islands, J. Appl. Phys. 98, 033530 (2005).

[39] A. V. Baranov, A. V. Fedorov, T. S. Perova, R. A. Moore, V. Yam, D. Bouchier, V. Le Thanh, and K. Berwick, Analysis of strain and intermixing in single-layer $\mathrm{Ge} / \mathrm{Si}$ quantum dots using polarized Raman spectroscopy, Phys. Rev. B 73, 075322 (2006).

[40] A. Bernardi, M. I. Alonso, J. S. Reparaz, A. R. Goni, P. D. Lacharmoise, J. O. Osso, and M. Garriga, Evolution of strain and composition during growth and capping of Ge quantum dots with different morphologies, Nanotechnology 18, 475401 (2007).

[41] J. S. Reparaz, A. Bernardi, A. R. Goni, M. I. Alonso, and M. Garriga, Composition dependence of the phonon strain shift coefficients of SiGe alloys revisited, Appl. Phys. Lett. 92, 081909 (2008).

[42] Y. S. Chiu, M. H. Ya, W. S. Su, and Y. F. Chen, Properties of photoluminescence in type-II GaAsSb/GaAs multiple quantum wells, J. Appl. Phys. 92, 5810 (2002).

[43] M. Jo, M. Sato, S. Miyamura, H. Sasakura, H. Kumano, and I. Suemune, Origin of the blueshift of photoluminescence in a type-II heterostructure, Nano. Res. Lett. 7, 1 (2012). 